\title{
Native kidney small renal masses in patients with kidney transplants: Does chronic immunosuppression affect tumor biology?
}

Ari P. Bernstein ${ }^{1}$; Meenakshi Davuluri²; Matthew DeMasi ${ }^{1}$; Alexander Sankin²; Kara Watts²; Ahmed Aboumohamed ${ }^{2}$; Joshua M. Stern'ㄱ Juan Rocca ${ }^{3}$; Stuart Greenstein ${ }^{3}$; Jay A. Graham ${ }^{3}$; Maria Ajaimy ${ }^{3}$; Luz Liriano-Ward ${ }^{3}$; Judy Sarungbam ${ }^{4}$; Evan Kovac ${ }^{2}$

${ }^{1}$ Albert Einstein College of Medicine, New York, NY, United States; ${ }^{2}$ Department of Urology, Montefiore Medical Center, New York, NY, United States; ${ }^{3}$ Montefiore Einstein Center for Transplantation, New York, NY, United States; ${ }^{4}$ Department of Pathology, Montefiore Medical Center, New York NY, United States

Cite as: Bernstein AP, Davuluri M, DeMasi M, et al. Native kidney small renal masses in patients with kidney transplants: Does chronic immunosuppression affect tumor biology? Can Urol Assoc J 2021 March 18; Epub ahead of print. http://dx.doi.org/10.5489/cuaj.6996

Published online March 18, 2021

$* * *$

\begin{abstract}
Introduction: We compared clinicopathologic characteristics and outcomes of radical nephrectomy $(\mathrm{RN})$ for small renal masses $(\mathrm{SRM})$ in patients with end-stage renal disease (ESRD) before or after transplant at a high-volume urologic and transplant center.
\end{abstract}

Methods: We performed a retrospective review of patients with ESRD (glomerular filtration rate [GFR] $<15 \mathrm{~mL} / \mathrm{min}$ ) who underwent RN for suspected malignant SRM from 2000-2018. Group 1 consisted of patients who underwent RN after transplant; group 2 underwent RN prior to transplant, and group 3 underwent RN without subsequent transplant. Dominant tumor size and histopathologic characteristics, recurrence, and survival outcomes were compared between groups. Chi-squared and Mann-Whitney U tests were used to compare categorical and continuous baseline and histopathologic characteristics, respectively. Univariate analysis and log rank test were used to compare $\mathrm{RCC}$ recurrence rates.

Results: We identified 34 nephrectomies in group 1, 27 nephrectomies in group 2, and 70 nephrectomies in group 3. Median time from transplant to SRM radiologic diagnosis in group 1 was 87 months, and three months from diagnosis to nephrectomy for all groups. There were no statistically significant differences between pathologic dominant mass size, histologic subtype breakdown, grade, or stage between the groups. Rates of benign histology were similar between the groups. Univariate analysis did not reveal a statistically significant difference in recurrencefree survival between the groups $(p=0.9)$. 
Conclusions: Patients undergoing nephrectomy before or after transplant for SRM have similar indolent clinicopathologic characteristics and low recurrence rates. Our results suggest that chronic immunosuppression does not adversely affect SRM biology.

\section{Introduction}

The risk of developing native kidney renal cell carcinoma (RCC) is increased in patients with end stage renal disease (ESRD) undergoing dialysis and in patients that have received kidney transplants due to ESRD. ${ }^{1-3}$ Patients with ESRD and who develop native kidney small renal masses (SRM) that are suspicious for RCC are therefore often advised to undergo surgical resection based on the theoretical risk of impaired immune surveillance and subsequent disease progression while receiving chronic immunosuppression after kidney transplantation.

Recent landmark studies have revealed that RCC biology is highly responsive to its immune microenvironment, ${ }^{4}$ thus providing rationale for aggressive treatment of RCC in transplant candidates or recipients. However, for otherwise healthy patients, current guidelines recommend active surveillance (AS) for $\mathrm{SRM} \leq 3 \mathrm{~cm}$ in select patients as metastasis rates are extremely low in this population and up to $20 \%$ are benign. ${ }^{5}$ Nonetheless, few guidelines exist for management of SRM in patients with ESRD, in whom active malignancy is often considered a contraindication to transplantation. Currently, there is little evidence to support the association between immunosuppression and aggressive RCC tumor biology or disease progression. Therefore, newly detected SRM in ESRD patients that would otherwise be surveillance candidates are often treated with nephrectomy despite a relative lack of data supporting the need for expedited surgical intervention.

We therefore investigated whether there were any meaningful differences in both RCC histopathology and clinical outcomes after nephrectomy in pre-transplant, ESRD patients on dialysis versus patients who have received kidney transplants and chronic immunosuppression.

\section{Methods}

We performed a retrospective review of patients with CKD stage $\mathrm{V}$ and ESRD (glomerular filtration rate $<15 \mathrm{~mL} / \mathrm{min} / 1.73 \mathrm{~m}^{2}$ ) who underwent open or laparoscopic radical nephrectomy (RN) for native kidney SRM between 2000-2018 at a high volume kidney transplant institution. All patients with SRM were included in this study, regardless of location or clinical T-stage. SRM was defined as cT1 (i.e. $<7 \mathrm{~cm}$ ). Group 1 consisted of patients who underwent nephrectomy after transplant, regardless of pre-transplant kidney function; Group 2 consisted of patients with ESRD who underwent nephrectomy with subsequent transplantation, and Group 3 consisted of patients with ESRD who underwent nephrectomy without subsequent transplantation. Baseline demographics, including age at nephrectomy, sex and smoking history, were compared between the groups. We compared rates of benign pathological findings at nephrectomy and the following histopathologic characteristics of pathologically confirmed malignant tumors between the 
groups: malignant histology breakdown, dominant tumor (largest tumor) size (cm), pathologic stage and WHO/International Society of Urological Pathology (ISUP) nucleolar grade. Nephrometry scores were not calculated given that all patients underwent radical rather than partial nephrectomy. We then compared post-nephrectomy RCC recurrence-free survival rates between the groups.

Chi-square and Mann-Whitney $U$ tests were used to compare categorical and continuous baseline and histopathologic characteristics, respectively. Kruskall-Wallis test was used to compare Charlson Comorbidity Indices between groups. Fischer's exact test was used to evaluate differences between immunosuppressive regimes. Univariate analysis and log rank test were used to compare RCC recurrence rates between groups after nephrectomy and KaplanMeier method was used to generate recurrence-free survival curves. All statistical tests were performed using SPSS version 23 (IBM Corp. Armonk, NY).

\section{Results}

We initially identified 96 patients meeting criteria for group 1 (nephrectomy after transplant) and 422 patients meeting criteria for both groups 2 and 3 (nephrectomy with or without subsequent transplant, respectively) before evaluating for status of eventual transplantation. 65 and 335 patients were excluded from these initial two cohorts, respectively, due to nephrectomies performed for reasons other than suspected malignancy and/or patients not meeting our selective definition of CKD or ESRD $\left(\mathrm{GFR}<15 \mathrm{~mL} / \mathrm{min} / 1.73 \mathrm{~m}^{2}\right)$. After further dividing patients into three groups, we identified 34 nephrectomies (in 31 patients) in group 1, 27 nephrectomies (in 24 patients) in group 2, and 70 nephrectomies (in 63 patients) in group 3 meeting final inclusion criteria (Figure 1). 8 (24.0\%), $3(11.0 \%)$, and 10 (14.0\%) SRM were pathologically benign in groups 1,2 , and 3 , respectively $(\mathrm{p}=0.4) .84(64 \%)$ surgical pathology specimens underwent central pathology re-review while 48 (36\%) were unavailable for central re-review. All surgical pathology margins were negative. Median number of distinct masses per kidney was 1 in all three groups. Median time from transplant and initiation of immunosuppression to nephrectomy in group 1 was 87 months (interquartile range [IQR] 42-134). Median time from nephrectomy to transplant in group 2 was 24 months (IQR 7-54). No patients in this study underwent simultaneous nephrectomy and transplant. Median time from radiologic SRM diagnosis to nephrectomy was 3 months for all groups (IQR 2-7).

Baseline demographic and clinical features of both groups are depicted in Table 1. Patients in Group 2 were significantly younger and Group 3 patients had higher Charlson Comorbidity Indices. Most patients in Group 2 received T-cell depleting (rabbit anti-thymocyte globulin) induction immunosuppression as compared to IL-2 receptor antagonists (20 vs. 6 , respectively); this is in comparison to Group 1 which received more IL-2 receptor antagonist therapy than T-cell depleting immunosuppression (16 vs. 12, respectively). More patients in group 2 underwent maintenance immunosuppression with mycophenolate mofetil, though no other differences between maintenance immunosuppressive regimens were noted between group 
$1 \& 2$ (Table 1). Mean duration of dialysis prior to transplant was 5 years and 2 months in group 1. Mean duration of dialysis prior to nephrectomy was 9.4 years and 8.2 years in group 2 and 3 respectively.

Histopathological features of the pathologically confirmed malignant SRMs are depicted in Table 2. There were no statistically significant differences between pathologic dominant mass size, benign histologic rate, malignant histologic subtype breakdown, grade or stage between the groups. Sarcomatoid features were only identified in 1 patient with clear cell RCC in group 1.

Median follow-up after nephrectomy was 40, 49, and 33 months for groups 1, 2, and 3, respectively $(\mathrm{p}=0.01)$. There were no differences in the number of scans after nephrectomy between the groups (Table 1). Figure 2 depicts radiographic recurrence-free survival outcomes between patients with malignant histology in all three groups. Three patients recurred (all in group 3); two had acquired cystic disease-associated RCC in the primary that metastasized to the liver and pelvic bone, and one had papillary type $2 \mathrm{RCC}$ in the primary that later presented with bilateral renal masses. Among the three patients with recurrent disease, one patient was treated with a tyrosine kinase inhibitor (pazopanib), another with radiation therapy alone, and the third patient received unknown treatment, which occurred at an outside hospital. Notably, recurrence was absent in groups 1 and 2. No difference in recurrence rates were found between groups 1 and $3(p=0.19)$ as well as between groups 2 and $3(p=0.17)$. When combining groups 1 and 2 (immunosuppressed) and comparing it to group 3 (non-immunosuppressed), there was no significant difference in recurrence rate $(\mathrm{p}=0.06)$. Univariate analysis did not reveal a statistically significant difference in recurrence-free survival between the groups (Log-Rank $=0.2$ for all pairwise comparisons). Three and 13 patients died in groups 1 ( 1 due to sepsis; 2 unknown) and 3 (4 due to sepsis, 4 due to cardiac arrest, and 5 unknown), respectively. No patients died in group 2. No patients died of RCC progression during the follow up period.

\section{Discussion}

In our study of patients with ESRD, post-kidney transplant patients who receive chronic immunosuppression and who undergo nephrectomy for SRM have similar rates of benign histology, similar malignant pathologic characteristics and similar radiographic recurrence-free survival rates compared to pre-transplant patients who are not chronically immunosuppressed. Though our study included cT1 masses up to $7 \mathrm{~cm}$, the vast majority of masses included were $<3 \mathrm{~cm}$. Overall, we believe our findings call into question the practice of mandatory nephrectomy for pre-transplant patients due to the theoretical heightened risk of RCC progression while on chronic immunosuppression after kidney transplantation. These findings suggest that we may be unnecessarily delaying transplants and subjecting certain patients to non-emergent surgery.

The practice of early surgical intervention for suspected malignant SRM in this patient population is rooted in the demonstrated link between immunosuppression and carcinogenesis. Previous studies have concluded that the incidence of malignancy is higher in solid organ transplant recipients compared with age-matched counterparts in the general population and that 
increased length and intensity of immunosuppressive therapy directly correlates with tumor aggression and is inversely related to survival outcomes. ${ }^{6}$ While the exact biologic mechanism(s) underlying this epidemiological relationship have yet to be elucidated, the overarching theory is that of impaired immunosurveillance, leading to both unchecked oncogenic mutations and facilitation of the natural action of pro-oncogenic viruses to which immunosuppressed patients are already prone. ${ }^{7}$ Another purported theory is that immunosuppressive drugs may potentiate some environmental carcinogens such as sunlight, radiation and tobacco smoke. ${ }^{8}$ In a large comparative study of skin cancer incidence in transplant versus control patients, the transplant group demonstrated significantly more aggressive behavior as measured by markers of aggression including: recurrence rates, lymph node invasion and death from the disease. ${ }^{9}$

We compared several clinical and pathologic indicators of RCC aggressiveness between our groups, including rates of benign histology, malignant tumor subtype, grade, stage and radiographic recurrence. No statistically significant differences were observed among any of these biomarkers between cohorts. In addition, no patients died of renal cell carcinoma during a median follow-up of 40, 49 and 33 months after nephrectomy for groups 1, 2, and 3, respectively.

While the development of native kidney RCC in patients with ESRD is relatively common, few studies have directly examined the differences in tumor biology and clinical outcomes after nephrectomy between pre and post-transplant patients. A 2018 meta-analysis of patients with a history of urologic malignancies who then underwent renal transplant found that renal transplantation did not increase the risk of $\mathrm{RCC}$ recurrence relative to patients who remained on dialysis, ${ }^{10}$ consistent with our findings. Conversely, a study of 202 Japanese patients compared native kidney RCC outcomes between renal transplant recipients versus dialysis patients and found increased RCC recurrence rates and decreased survival outcomes in the transplant group. ${ }^{11}$ However, both of the above mentioned studies only included patients who were surgically treated for RCC prior to transplantation and did not specifically compare pathologic and clinical outcomes of de novo RCC that developed before and after renal transplantation. Therefore, while immunologic translational data suggests a potential for more aggressive RCC biology in the immunosuppressed state, these adverse pathologic and clinical phenotypes are not clinically borne out of our study.

Our study contributes to the growing body of literature on surgical outcomes of malignant diseases in transplant patients who are chronically immunosuppressed and specifically challenges the current dogma of removing all SRM in patients who are either transplant candidates or recipients. Current European guidelines state that kidney transplant recipients who have tumors with low recurrence rates can be considered for immediate transplantation after successful treatment of the tumor. However, our findings suggest that a period of surveillance may be an option for pre- and post-transplant patients with SRM. ${ }^{12}$ The reason we believe this is based on our study results; since most patients exhibited excellent recurrence-free survival rates 
after nephrectomy, and clinical and pathologic indicators of RCC aggressiveness and tumor biology were similar between ERSD patients with and without immunosuppression, then by extension, immediate transplantation with a short course of surveillance may be an appropriate response for pre-transplant patients with SRM. Additionally, with a median wait time for kidney transplantation in the U.S. of 4.0 years, further investigation of clinical outcomes in transplant patients with SRM is of significant clinical importance when the survival benefits to transplantation over dialysis are so clearly documented in the literature, and avoidance of delayed transplantation is paramount. ${ }^{13}$ As our study has also shown that a larger percentage of dialysis patients develop acquired cystic disease-associated RCC relative to the post-transplant cohort, delaying transplant and remaining on dialysis could have detrimental impacts. Therefore, we believe transplant may not need to be delayed for nephrectomy, and that a short course of surveillance for the SRM may be a viable option.

For patients amenable to AS, rather than immediate nephrectomy, the goal is to surgically intervene when clinically appropriate and prior to local progression or distant metastatic spread. Patients are generally followed according to established guidelines, ${ }^{14}$ and include an abdominal computed tomography (CT) or magnetic resonance imaging (MRI) within 6 months of initiating $\mathrm{AS}$, and yearly thereafter. Additionally, an annual Chest X-ray (CXR) is recommended for those with biopsy proven RCC or a tumor with oncocytic features. Clinical indications for intervention while on AS vary by institution, but patients with SRM that demonstrate accelerated growth velocity on serial imaging or that grow beyond $3 \mathrm{~cm}$ are generally recommended to undergo surgical intervention.

In the post-nephrectomy setting, continued surveillance for recurrence is risk-stratified and tailored according to the clinical risk of recurrence. For low-risk patients (pT1, N0, Nx), guidelines recommend abdominal imaging (ultrasound [US], CT, or MRI) within 3-12 months following surgery, as well as an annual CXR for three years. Moderate or high-risk patients (pT2-4N0, Nx or any $\mathrm{N}+$ ) typically undergo a baseline abdominal CT or MRI, as well as a baseline chest CT, within 3-6 months following surgery, as well as continued imaging (abdominal US, CT, or MRI; chest CT or CXR) every 6 months for at least 3 years, and annually to year $5 .{ }^{14}$

We found that pathologic markers of aggressive biology (high-grade, stage $\geq \mathrm{pT} 3$, sarcomatoid features) were generally low in all groups. Recurrence rates were also low (only 3 total, all in group 3) and do not significantly differ from large series of SRM excisions in the non-immunosuppressed. ${ }^{15,16}$ Furthermore, $\mathrm{RN}$ is not without risk, especially in this population. Complications such as bleeding, infection, bowel injury, liver injury, pancreatic injury, pneumothorax, venous thromboembolism and even death have been reported after open and laparoscopic RN. ${ }^{17-19}$ Similarly, biopsy as a means of diagnosing malignant SRM remains controversial and is not mainstay for transplant patients specifically, though recent studies have demonstrated improved predictive value and perhaps there exists a role in management of SRMs 
in such patients. ${ }^{20}$ Despite the need for frequent surveillance imaging and the potential increased exposure to ionizing radiation ${ }^{21}$ and nephrotoxic contrast media ${ }^{22}$ for those on AS, surveillance has demonstrated to be the most cost-effective option for patients with small renal masses and the only option to avoid the risks of general anesthesia and radical nephrectomy. ${ }^{23}$ Our results therefore suggest that transplantation could perhaps be performed in dialysis patients presenting with an SRM prior to nephrectomy and that AS might be a reasonable option in this patient population. Importantly, urologic oncologists must take into account that in select, healthy Group 1 patients the risk of nephrectomy is quite low whereas consequences of possible RCC metastasis is very significant. This risk versus benefit evaluation must be made on a patientspecific basis, especially in the context of patients with newly transplanted functioning allograft kidneys harboring native, nonfunctional kidneys with a renal mass of metastatic potential, even if low. Reconsideration of nephrectomy for SRM in the native kidney should therefore take place after successful transplantation in clinically stable patients who may have elected for AS of the renal mass prior to transplantation. Importantly, evaluation of urine output from the native kidney should be included in the decision making process for nephrectomy, with anuric kidneys more likely warranting removal versus those with urine production.

Our study does carry several limitations. The retrospective design of our study lends itself to inherent selection bias. Additionally, our ethnically diverse patient in the Bronx may not approximate other regional populations. Our grouping of patients into three distinct groups may inherently contribute to selection bias that impacts our ultimate findings. The lack of negative events noted in groups 1 and 2 may be directly related to small sample sizes in these groups. Similarly, although differences did not exist in tumor recurrence, induction immunosuppression may be relevant to tumor behavior with evidence showing T-cell depleting induction to be superior to IL-2 antagonist therapy in high risk patients; ${ }^{24,25}$ in our cohort of patients, T-cell depleting induction was found in a greater proportion of Group 2 patients than those of Group 1. Finally, while our sample size is relatively small, we believe this is the first study to directly compare the outcomes of pre and post-renal transplant recipients where the effects of chronic immunosuppression on RCC biology and outcomes are largely unknown. We believe our results are hypothesis generating and support the need for larger, multi-institutional randomized studies. In addition, we believe that our findings contribute significant understanding of the surgical outcomes of SRM excision in ESRD patients and increase our knowledge of the link between immunosuppression and RCC-specific pathology and surgical outcomes.

\section{Conclusions}

Patients undergoing radical nephrectomy before or after kidney transplant with suspected malignant native kidney SRM notably have similar clinicopathologic characteristics and recurrence-free survival outcomes. Thus, tumor biology and pathologic characteristics do not seem to change with immunosuppression. Since ESRD and dialysis treatment has been shown to be possibly detrimental, with increased rates of acquired cystic-disease associated RCC as shown 
in our study, and with poorer survival benefits as compared to transplant, we believe that a SRM in and of itself should not delay transplant. Because surveillance is now the standard for SRM due to their well-established indolent biology and low metastatic potential, and since the SRMs in both our pre- and post-transplantation study populations demonstrate similarly indolent biological characteristics, we believe our results suggest that surveillance might be a viable option in ESRD patients who are either awaiting transplantation or have received renal transplantation and immunosuppression.

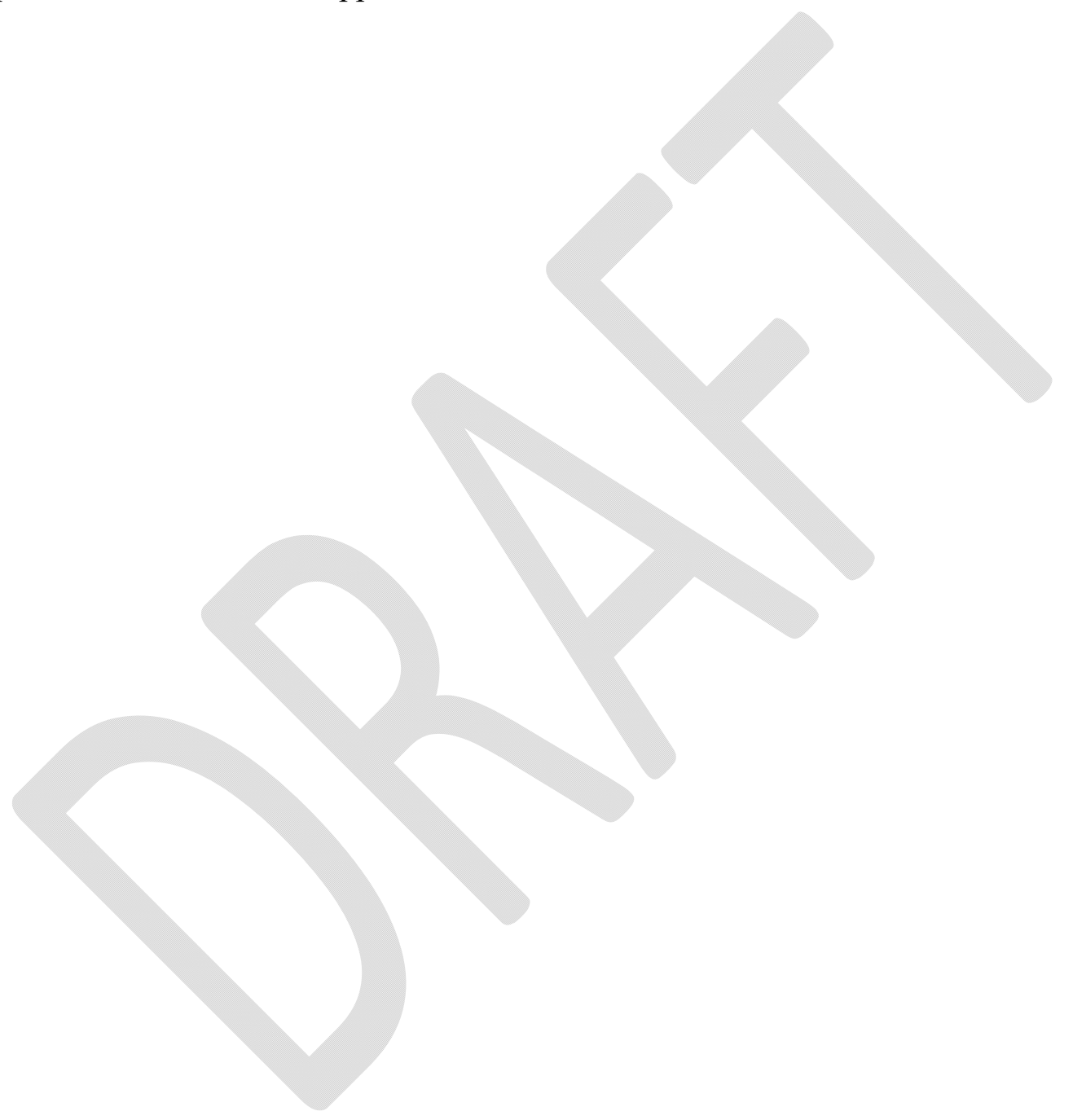




\section{References}

1. Birkeland SA LH, Storm HH. Cancer risk in patients on dialysis and after renal transplantation. Lancet 2000;355:1886-87.

2. Russo P. End Stage and Chronic Kidney Disease: Associations with Renal Cancer. Front Oncol 2012;2.

3. Zorbas KA KS, Lau KN, Di Carlo A. Renal Cell Carcinoma in Kidney Transplant Candidates. Transplant Proc 2017;49:1312-17.

4. Chevrier S LJ, Zanotelli VRT, Silina K, et al. An Immune Atlas of Clear Cell Renal Cell Carcinoma. Cell 2017;169:736-49.

5. Campbell SC NA, Belldegrun A, Blute ML, et al. Guideline for management of the clinical T1 renal mass. J Urol 2009;182:1271-79.

6. Gutierrez-Dalmau A CJ. Immunosuppressive therapy and malignancy in organ transplant recipients: a systematic review. Drugs 2007;67:1167-98.

7. Vial T DJ. Immunosuppressive drugs and cancer. Toxicology 2003;185:229-40.

8. $\quad$ Penn I ST. Immunosuppression and cancer. Transplant Proc 1973;5:943-47.

9. Lott DG MR, Koch C, Lorenz RR. Aggressive behaviors of nonmelanotic skin cancers in solid organ transplant recipients. Transplantation 2010;90:683-87.

10. Boissier R HV, Bruines HM, Budde K, et al. The risk of tumour recurrence in patients undergoing renal transplantation for end-stand renal disease after previous treatment for a urological cancer: a systematic review. European Urology 2018;73:94-108.

11. Ryosaka M IH, Takagi T, Shimizu T, et al. Solid-type RCC originating from native kidneys in renal transplant recipients should be monitered cautiously Transpl Int 2015;28:813-19.

12. Rodriguez Faba O BR, Budde K, et al. European Association of Urology Guidelines on Renal Transplantation: Update 2018. Eur Urol Focus 2018;4:208-15.

13. Mathur AK AV, Sands RL, Wolfe RA. Geographic Variation in End-Stage Renal Disease Incidence and Access to Deceased Donor Kidney Transplantation. Am J Transplant 2010;10:1069-80.

14. Follow-up for Clinically Localized Renal Neoplasms. American Urological Association. Guidelines: Renal Cancer: Follow-up for Clinically Localized Renal Neoplasms Guideline Web site. https:/www.auanet.org/guidelines/renal-cancerfollow-up-for-clinically-localized-renal-neoplasms-guideline. Published 2013. Accessed.

15. Lane BR GI. 5-Year outcomes of laparoscopic partial nephrectomy. J Urol 2007; 177:70-74.

16. Lee CT KJ, Shi W, Thaler HT, et al. Surgical management of renal tumors $4 \mathrm{~cm}$ or less in a contemporary cohort. J Urol 2000;163:730-36.

17. Kim FJ RK, Hernandez F, Jarrett TW, et al. Laparoscopic radical versus partial nephrectomy: assessment of complications. J Urol 2003;170:408-11.

18. Shekarriz B UJ, Shekarriz H, de Assis Mendes Goes F Jr, et al. Comparison of costs and complications of radical and partial nephrectomy for treatment of localized renal cell carcinoma. Urology 2002;59:211-15. 
19. Stephenson AJ HA, Snyder ME, Russo P. Complications of radical and partial nephrectomy in a large contemporary cohort. J Urol 2004;171:130-34.

20. Marconi L DS, Lam TB, et al. Systematic Review and Meta-analysis of Diagnostic Accuracy of Percutaneous Renal Tumour Biopsy. European Urology 2016;69:66073.

21. Oh JS, Koea JB. Radiation risks associated with serial imaging in colorectal cancer patients: should we worry? World J Gastroenterol 2014;20:100-09.

22. Andreucci M, Solomon R, Tasanarong A. Side effects of radiographic contrast media: pathogenesis, risk factors, and prevention. BioMed research international 2014;2014:741018.

23. Chang SL, Cipriano LE, Harshman LC, et al. Cost-effectiveness analysis of nephron sparing options for the management of small renal masses. J Urol 2011;185:1591-7.

24. KDIGO clinical practice guideline for the care of kidney transplant recipients. Am $J$ Transplant 2009;9 Suppl 3:S1-155.

25. Brennan DC, Daller JA, Lake KD, et al. Rabbit Antithymocyte Globulin versus Basiliximab in Renal Transplantation. New England Journal of Medicine 2006;355:1967-77.

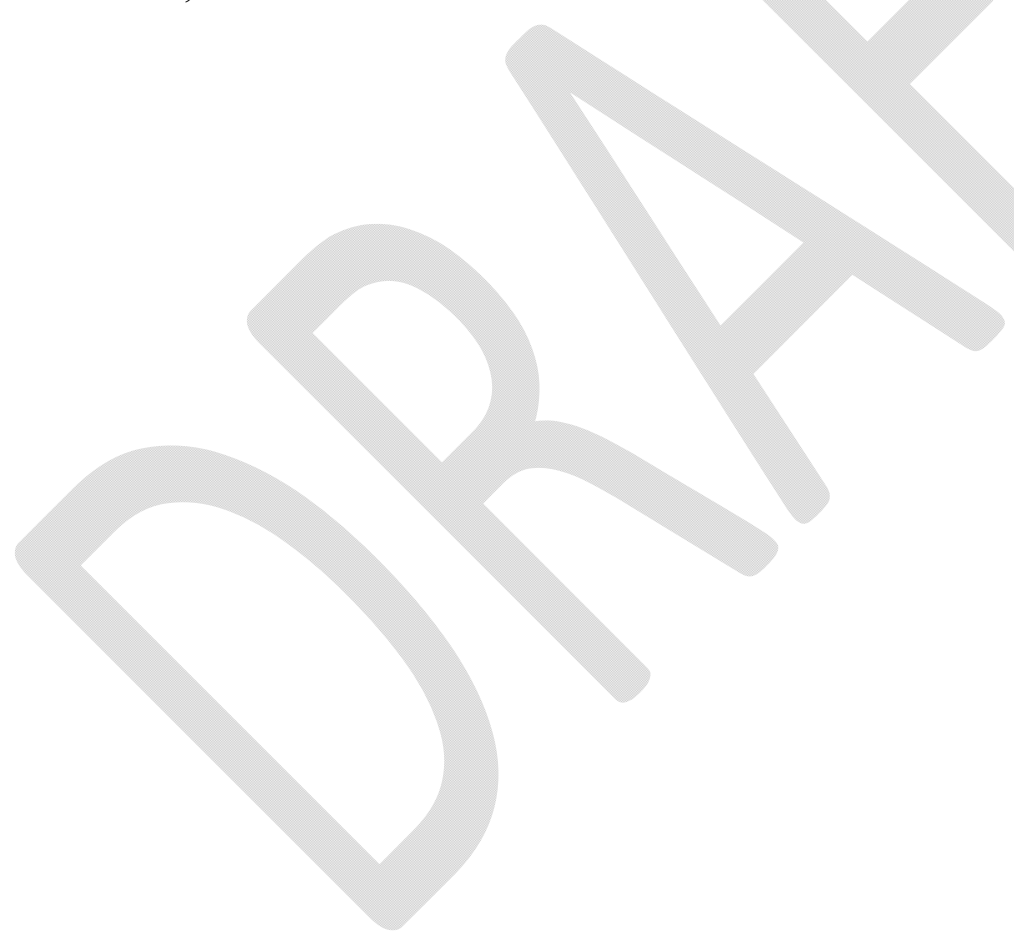




\section{Figures and Tables}

Fig. 1. Study flowchart. eGFR: estimated glomerular filtration rate; ESRD: end-stage renal disease.
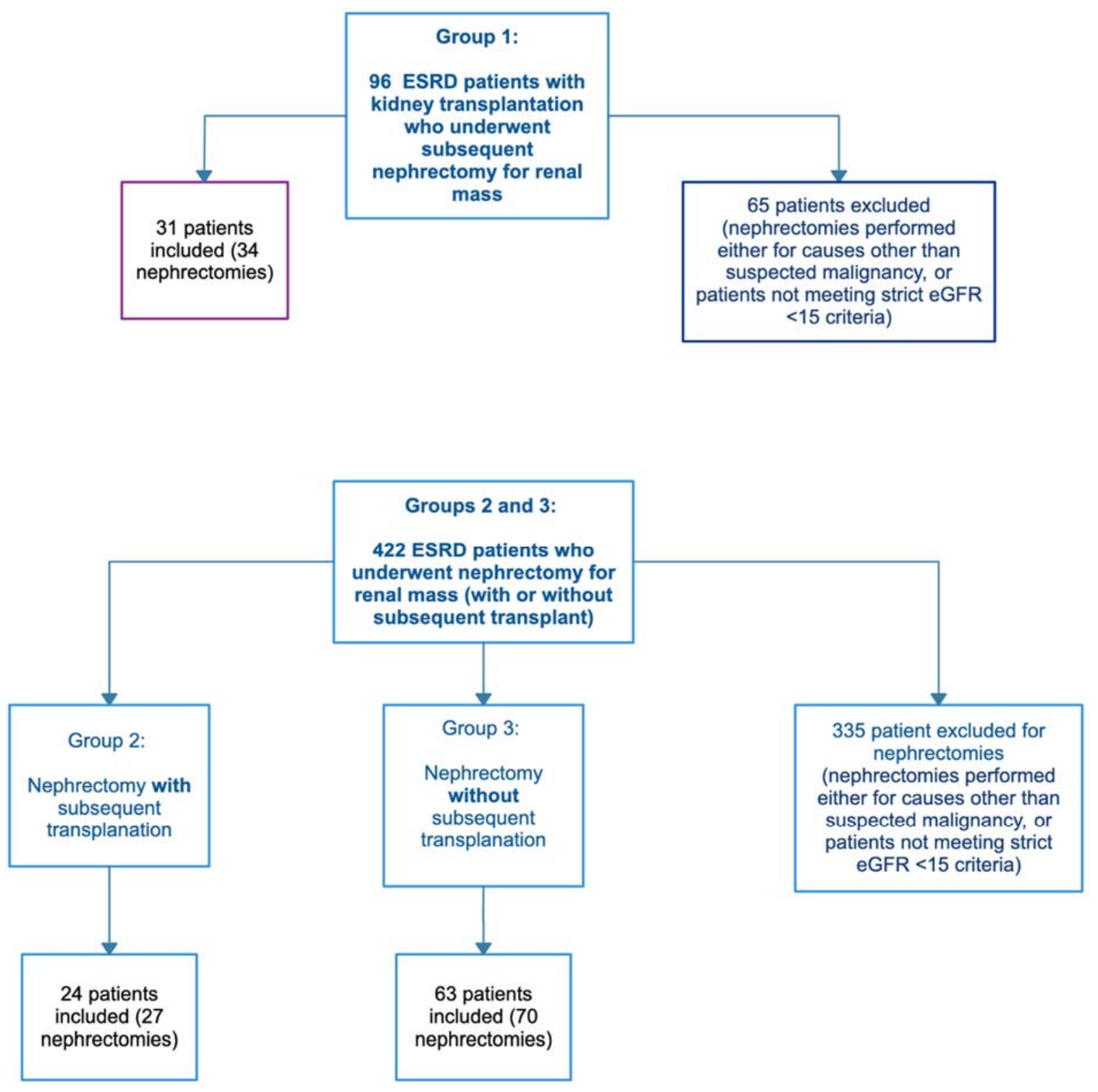
Fig. 2. Radiographic recurrence-free survival outcomes between patients with malignant histology in all three groups.

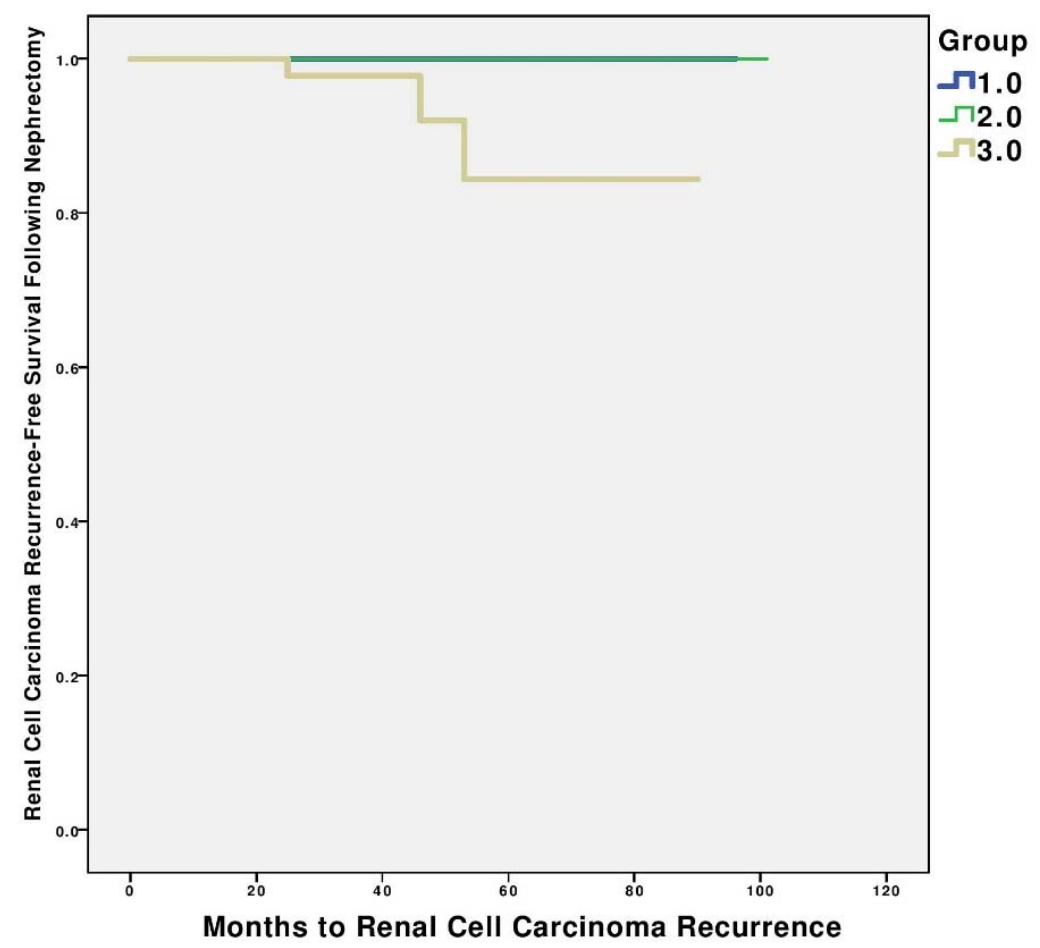


Table 1. Baseline features of groups 1, 2, and 3 patients

\begin{tabular}{|c|c|c|c|c|}
\hline Characteristics & $\begin{array}{c}\text { Group 1: } \\
\text { Nephrectomy } \\
\text { after transplant }\end{array}$ & $\begin{array}{c}\text { Group 2: } \\
\text { Nephrectomy } \\
\text { followed by } \\
\text { transplant }\end{array}$ & $\begin{array}{c}\text { Group 3: } \\
\text { Nephrectomy but } \\
\text { no transplant }\end{array}$ & $\mathbf{p}$ \\
\hline Nephrectomies, n (individual patients) & $34(31)$ & $27(24)$ & $70(63)$ & \\
\hline Median age at nephrectomy (IQR) & $60(54,68)$ & $47(46,53)$ & $59(53,68)$ & $<\mathbf{0 . 0 1}$ \\
\hline Sex, n $(\%)$ & & & & 0.9 \\
\hline Female & $13(38)$ & $11(41)$ & $27(39)$ & \\
\hline Male & $21(62)$ & $16(59)$ & $43(61)$ & \\
\hline Race, $\mathrm{n}(\%)$ & & & & $<0.01$ \\
\hline White & $1(3)$ & $9(33)$ & $8(11)$ & \\
\hline Black & $18(53)$ & $7(26)$ & $48(69)$ & \\
\hline Hispanic & $11(32)$ & $9(33)$ & $10(14)$ & \\
\hline Asian & $3(9)$ & $0(0)$ & $1(1)$ & \\
\hline Other/unknown & $0(0)$ & $2(7)$ & $3(4)$ & \\
\hline $\begin{array}{l}\text { Median Charlson comorbidity index } \\
\text { (IQR) }\end{array}$ & $6(5,7)$ & $5(4,6)$ & $7(5,8)$ & 0.002 \\
\hline Hypertension, n (\%) & $32(94)$ & $25(93)$ & $65(93)$ & 0.8 \\
\hline Diabetes mellitus, m (\%) & $11(32)$ & $14(52)$ & $22(31)$ & 0.2 \\
\hline \multicolumn{5}{|l|}{ Induction immunosuppression, n (\%) } \\
\hline T-cell depleting therapy & $12(35)$ & $20(74)$ & - & 0.01 \\
\hline IL-2 Induction & $16(47)$ & $6(22)$ & - & 0.01 \\
\hline \multicolumn{5}{|l|}{ Maintenance immunosuppression, n (\%) } \\
\hline Tacrolimus & $25(74)$ & $25(93)$ & - & 0.08 \\
\hline Mycophenolate mofetil & $17(50)$ & $27(100)$ & - & $<0.001$ \\
\hline Cyclosporine & $6(18)$ & $2(7)$ & - & 0.2 \\
\hline Prednisone & $33(97)$ & $26(96)$ & - & 0.5 \\
\hline Other & $3(9)$ & $0(0)$ & - & 0.2 \\
\hline Smoking history, n (\%) & $15(44)$ & $16(59)$ & $34(49)$ & 0.5 \\
\hline $\begin{array}{l}\text { Median followup after nephrectomy, } \\
\text { months (IQR) }\end{array}$ & $40(29,52)$ & $49(36,89)$ & $33(18,49)$ & 0.01 \\
\hline
\end{tabular}


Median number of scans after nephrectomy (IQR)

IQR: interquartile range.
$1(0.25,3)$

$3(2,5)$

$2(1,4)$

0.07

\begin{tabular}{|c|c|c|c|c|}
\hline Characteristics & $\begin{array}{c}\text { Group 1: } \\
\text { Nephrectomy after } \\
\text { transplant }(n=34)\end{array}$ & $\begin{array}{c}\text { Group 2: } \\
\text { Nephrectomy followed } \\
\text { by transplant }(n=27)\end{array}$ & $\begin{array}{c}\text { Group 3: } \\
\text { Nephrectomy but no } \\
\text { transplant }(n=70)\end{array}$ & $\mathbf{p}$ \\
\hline Benign (\%) & $8(24)$ & $3(11)$ & $10(14)$ & 0.4 \\
\hline Malignant (\%) & $26(76)$ & $24(89)$ & $60(86)$ & \\
\hline \multicolumn{5}{|l|}{$\begin{array}{l}\text { Malignant pathologic } \\
\text { histology breakdown, } \\
\mathrm{n}(\%)\end{array}$} \\
\hline Clear-cell & $9(35)$ & $3(13)$ & $9(15)$ & 0.1 \\
\hline Papillary type 1 & $9(35)$ & $5(21)$ & $8(13)$ & \\
\hline Papillary type 2 & $1(4)$ & $0(0)$ & $1(2)$ & \\
\hline Chromophobe & $1(4)$ & $0(0)$ & $1(2)$ & \\
\hline Unclassified & $1(4)$ & $2(8)$ & $1(2)$ & \\
\hline $\begin{array}{l}\text { Acquired cystic } \\
\text { disease-associated } \\
\text { RCC }\end{array}$ & $4(15)$ & $10(42)$ & $25(42)$ & \\
\hline Clear-cell papillary & $1(4)$ & $4(17)$ & $14(23)$ & \\
\hline Metastasis & $0(0)$ & $0(0)$ & $1(2)$ & \\
\hline $\begin{array}{l}\text { Median path max } \\
\text { dimension } \\
\text { of dominant mass }(\mathrm{cm}) \\
(\mathrm{IQR})\end{array}$ & $2.4(1.4-2.8)$ & $2.0(1.4-2.5)$ & $2.2(1.6-2.9)$ & 0.07 \\
\hline $\begin{array}{l}\text { Path stage breakdown, } n \\
(\%)\end{array}$ & & & & 0.7 \\
\hline $\mathrm{T} 1 \mathrm{a}$ & $23(88)$ & $22(92)$ & $43(72)$ & \\
\hline $\mathrm{T} 1 \mathrm{~b}$ & $2(8)$ & $1(4)$ & $10(17)$ & \\
\hline $\mathrm{T} 2 \mathrm{a}$ & $0(0)$ & $0(0)$ & $0(0)$ & \\
\hline $\mathrm{T} 2 \mathrm{~b}$ & $0(0)$ & $0(0)$ & $1(2)$ & \\
\hline T3a & $1(4)$ & $1(4)$ & $5(8)$ & \\
\hline
\end{tabular}




\begin{tabular}{|c|c|c|c|c|}
\hline Metastasis & $0(0)$ & $0(0)$ & $1(2)$ & \\
\hline $\begin{array}{l}\text { WHO/ISUP nucleolar } \\
\text { grading, } \mathrm{n}(\%)\end{array}$ & & & & 0.3 \\
\hline Low (1-2) & $12(46)$ & $15(63)$ & $24(40)$ & \\
\hline High (3-4) & $4(15)$ & $4(17)$ & $17(28)$ & \\
\hline Not reported & $10(38)$ & $5(21)$ & $19(32)$ & \\
\hline
\end{tabular}

ISUP: International Society of Urological Pathology; IQR: interquartile range; RCC: renal cell carcinoma; WHO: World Health Organization. 